\title{
Intelligent Fish Feeding Regime System using Vibration Analysis
}

\author{
M. A. Adegboye ${ }^{1 *}$, A. M. Aibinu ${ }^{2}$, J. G. Kolo ${ }^{3}$, T. A. Folorunso ${ }^{4}$, I. Aliyu ${ }^{5}$, \\ and Lee Sun $\mathrm{Ho}^{6}$ \\ ${ }^{1}$ Dept. of Computer Engineering, Federal University Oye-Ekiti, Ekiti, Nigeria \\ ${ }^{2,4}$ Dept. of Mechatronic Engineering, Federal University of Technology, Minna, \\ Nigeria \\ ${ }^{3}$ Dept. Computer Engineering, Federal University of Technology, Minna, Nigeria \\ ${ }^{5,6}$ Chonnam National University, 50 Daehak-ro, Yeosu, Jeollanam-do, 59626, Korea \\ ${ }^{1}$ mutiu.adegboye@fuoye.edu.ng, ${ }^{2}$ maibinu@gmail.com, ${ }^{3}$ jgkolo@futminna.edu.ng, \\ ${ }^{4}$ funso.taliha@futminna.edu.ng, ${ }^{5}$ ibal2010@yahoo.com, ${ }^{6}$ sunnoa@naver.com
}

\begin{abstract}
Aquaculture represents an important food production system with high quality protein for human consumption. The contributions of aquaculture to the world's total fish production cannot be over emphasized, however, however, feeding is the major challenges facing in aquaculture system. Thus, to address this, development of intelligent fish feeding regime system based on vibration analysis is proposed in this paper. This was accomplished with the use of a novel 8-directional Chain Code generator algorithm developed for the extraction of signals from accelerometer for the escape and feeding activities. For the escape activity, $x$-and $z$ coordinate were selected, while $x$ - and $y$-coordinate were chosen for the feeding behavioural activity. The choice of coordinate selects is based on the fact that escape activity exists more between $x$ - and $z$-coordinate, while feeding activity more exists between $x$-and $y$-coordinate. The set of sequence features obtained was further processed using Discrete Fourier Transform in analysing the movement boundary. The results obtained shows that developed classifier using Fourier Descriptors obtained from Chain Code is more sufficient for the recognition of different movement patterns than Fourier Descriptors obtained from movement boundary.
\end{abstract}

Keywords: Accelerometer, Aquaculture, Chain code, Feeding, Fish

\section{Introduction}

Demand for aquaculture products is increases worldwide [1]. Despite the continuous increase in demand for fish consumption, the rate of fish supply to meet up with demand remain a serious challenge[2]. A sizeable fish cultivation that can meet up with the demand is expected through the vertical and horizontal extension of the aquaculture practice. This would largely depend on the fish farm management and proper aquaculture development [3]. The phenomenon of overfeeding has become predominant in aquaculture sector owning to the inefficient management of feed [4]. One of the causes of this challenge is attributed to the inefficiency handling of feeding system [5]. Feed management that can meet up with fish demand is very important for abundant income intensification. The cost of feeding fish amount to $40-50 \%$ of

Article history:

Received (July 6, 2019), Review Result (August 11, 2019), Accepted (October 29, 2019) 
the total aquaculture operational cost [6]. Whereas about $60 \%$ of the food dispense into aquarium end up as particulates [7]. These accumulated particulates result to water pollution which further used oxygen to decompose and produce ammonia-nitrogen and other toxic substances that can harm the fish growth.

The amount of feed dispenses to match fish appetite plays significant roles in increasing fish productivity, while measuring of actual feed fish intake remain critical challenge [8]. Fish feeding based on regulatory frequency is often faced with specific time regulation. Hence, it is important to feed based on needs. Overfeeding can result to wastage of feed which might affect quality of the water and finally result to diseases and stress [9]. Apart from feed wastage, too much of feed can caused shortage of oxygen in the water most especially during hot weather. This can further lead to the higher biological oxygen demand and slows down the growth rate of fish and eventually reducing productivity and profitability efficiency [10]. Most of the existing automatic feeding regime system were designed to operate at pre-set time. However, the use of pre-set has been shown that as the feeding habit of fish changes based on growth rate, age, size or weather condition the quantities of feed schedule does not vary in accordance with these factors. Thus, continuous operation of such feeder may deposit too much of feed which may lead to water contamination, poor fish growth rate and eventually result to low productivities. Therefore, in order to maintain efficient feeding in aquaculture, it is necessary to develop an intelligent feeding regime system. Hence, this research work employed the application of fish movement pattern and proficient of computational intelligence for the automation of fish feeding regime system based on vibration analysis.

\section{Literature review}

Three major categories of fish feeding regime system namely; manual method of feeding, semi-automatic and automatics feeder are being in use. The manual method of feeding fish involves the use of techniques such as fish body weight per feed [11]; estimation of facial and metabolic waste output, feed conversation rate and bio-energetic data at different water temperatures [12]. This method seems to be finest means to manage amount feed fish intake as a result of user involvement. However, it is time consuming, labor intensive and not applicable in a large fish farm.

Digital timer and programmable timing are the major techniques widely used for the development of semi-automatic fish feeding regime system [13]. In [14], an automatic fish feeder using digital timer proposed. The timer configured using monostable and astable modes for precision timing circuits capable of generating precise oscillation or timing delay. In the monostable operation mode, capacitor network and single external resistor was used to control the time interval, while a one-short pulse generation and operation time was computed between 1 to 10 seconds in the astable mode. In [15]. A PIC microcontroller based fish feeder for efficient pellets distribution proposed. The system enables manual adjustment of feeding cycle and motor speed range suitable for the user based on size of the pond and pellets distribution. Automatic fish feeder using two different control for intensive mirror carp production to provide predetermined amounts of feed proposed in [16]. The first control is an opened loop control system based on AT89c51 controller to coordinate amount of dosage in an accordance to feeding timetable. While the second control system (closing loop) was designed to be regulated based on water temperature condition, fish weight and oxygen consumption. A recent research in [17] design automatic fish feeding system for soft shell crab using microcontroller. In the approach, $5 \%$ of the crab body weight was predefined to be dispense at every activation 
time to avoid food wastage. The results obtained shows difference of 0.05 to 0.1 gram between the predefined and amount of food dropped.

The application of prediction model for scenario prediction have been proposed in various studies in the research community [18]. In an effort to make the fish feeder to be intelligent, Computer Vision (CV) and Artificial Intelligent (AI) have been used to improve the performance of the system. An intelligent feeding regime system based on CV for indoor intensive culturing of eel proposed in [19]. The work aimed at determining fish appetite through the gathering behaviour of the eel using infrared photo electric sensor. The system was designed to revive at a specific period of time, failure of the sensor to sense the eel gathering for the three consecutive trials was defined to lead to feeder stoppage until next rouse. Thus, limits the risk of chance of high rate of feed wastage. However, this approach is not efficient and reliable as the system continues to operate based on the fish gathering without prior identification of the purpose. The optimal approach should be that the system is controlled based on the actual feed intake of the eel or ability to determine the purpose of gathering. More so, the system should only come up when the eels are in need of feed, not just after certain period of time.

Automatic fish feeding regime system based on eating behaviour of the fish in a highly dense aquaculture tank proposed by Atoum et al. [7]. The work aims at controlling amount of dispense meal in an optimal rate, thus improve the aquaculture production profit. The control strategy employed involved continuous decision on whether dispensed feeds are actually consuming via the detection of the amount of left over feed on the surface of the water using two methods: correlation filter to optimum local region within the video frame and computer vision machine based refinement to suppress identified feed. A fuzzy logic controller (FLC) system for providing fish with feed using linguistic estimations and rules for the daily feeding managements of sea bream larvae developed in [20]. A fuzzy logic based control system for fresh water aquarium proposed in [21]. The proposed system adopted data acquisition device for online parameter acquisition and analysis. FLC was used for input stress parameters controls such as temperature, dissolved oxygen and conductivity. However, the three measuring parameters adopted cannot alone determine the health status of the fish. Other parameters such as dissolved ammonia from fish excreta, waste feed and Carbon dioxide will ensure higher degree of accuracy. Also, the health status of the fish might not necessary represent the feeding consumption rate of the fish at a particular point in time. The optimal solution should be use of real time techniques that can determine the actual feeding behaviour of the fish.

\section{Methodology}

The datasets used in this study were obtained by Noda, et al. [22] using accelerometer aligned to fishes with the directions of the angular velocity as Gx; Gy; and Gz while that of acceleration is given as Ax; Ay; and Az also on x-, y- and z-coordinates respectively. In order to determine the feature vector that are suitable for the individual activity recognition, the fish movements directions were extracted using Freeman Chain Codes. For the escape activity, $x-$ and $\mathrm{z}$ - coordinate were selected, while $\mathrm{x}$ - and $\mathrm{y}$ - coordinate were chosen for the feeding behaviour activity. This choice of coordinate selects is based on the fact that escape activity transpires more between $\mathrm{x}$ - and $\mathrm{z}$ - coordinate, while feeding activity weightier between $\mathrm{x}$ - and $\mathrm{y}$ - coordinate. [Figure 1] (a) and (b) shows escape and feeding activities respectively. 


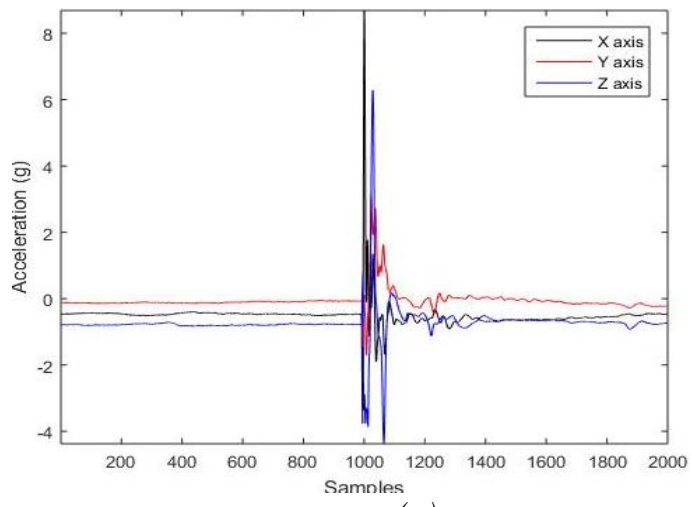

(a)

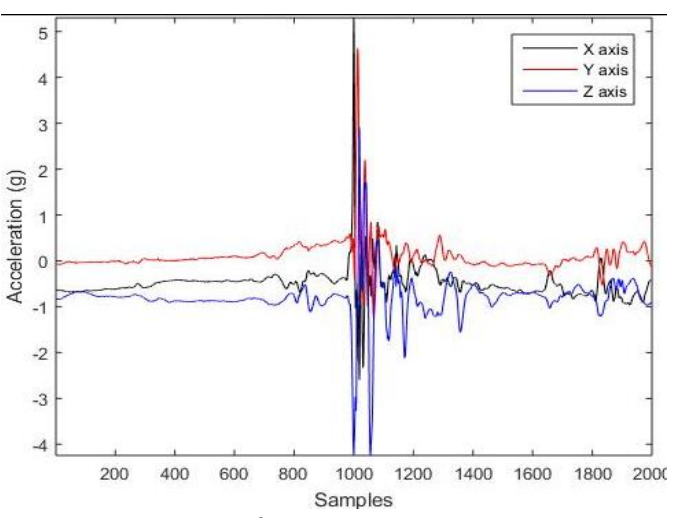

(b)

Figure 1. Accelerometer data plot for the (a) escape and (b) feeding movements

An 8-direction chain code was used to represent the fish movement direction through the connected sequence of straight-line segments of identified movement and direction. The 8directional Chain Code was chosen because it is more accurate than 4-connectivities Chain Code [23]. In this work, a novel 8-directional Chain Code algorithm was developed. The differences between the two points in each of the $\mathrm{x}-, \mathrm{y}$ - and $\mathrm{z}$ - coordinate were considered as delta $(\Delta)$. For computing the difference in the $\mathrm{x}$ - coordinate, the model used to obtain $\Delta \mathrm{x}$ is presented in (1). Conversely, (2) and (3) were used to compute the $\Delta y$ and $\Delta z$ in the $y-$ and zcoordinate respectively.

$$
\begin{aligned}
\Delta x & =x 2-x 1 \\
\Delta y & =y 2-y 1 \\
\Delta z & =z 2-z 1
\end{aligned}
$$

Where $\Delta \mathrm{x}, \Delta \mathrm{y}$ and $\Delta \mathrm{z}$ are the difference between the present and previous data point in the $\mathrm{x}-, \mathrm{y}-$ and $\mathrm{z}$ - coordinate respectively. The procedure for generating chain code is presented in Algorithm 1.

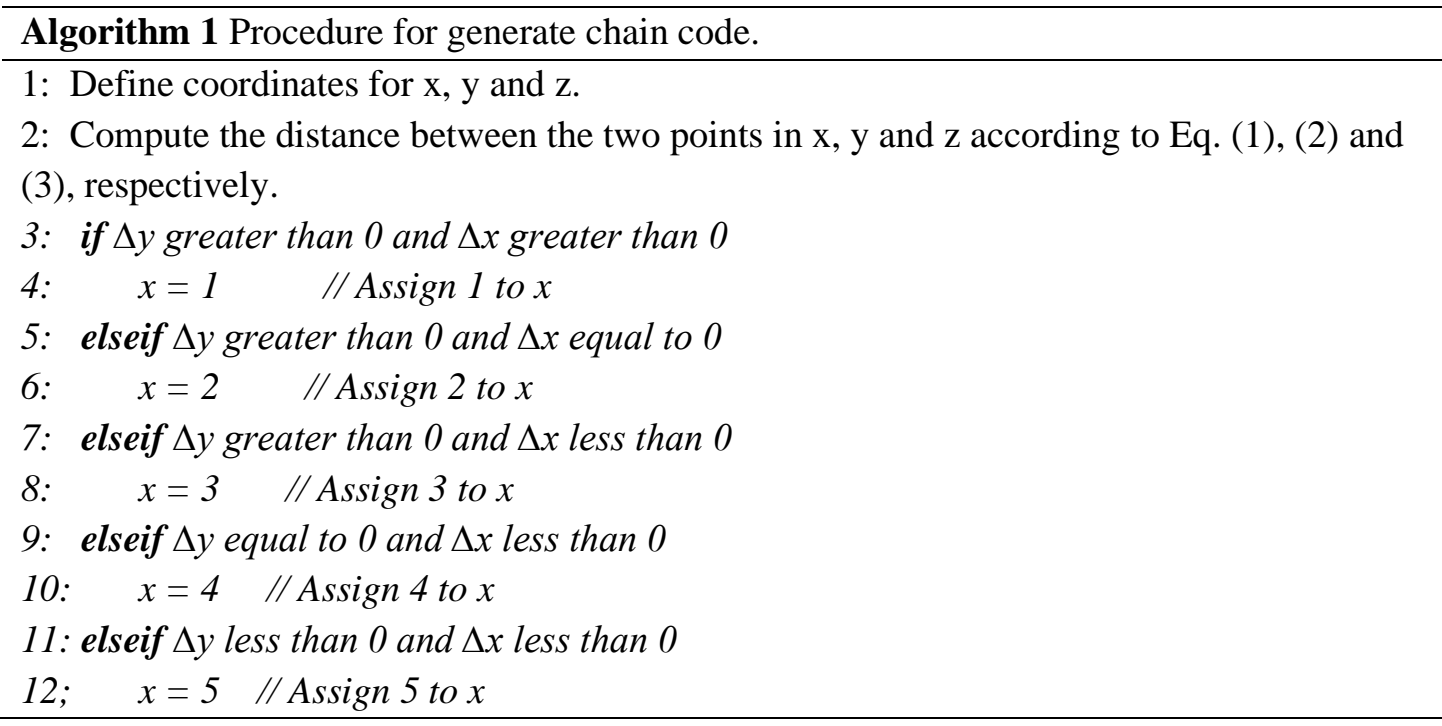


13: elseif $\Delta y$ less than 0 and $\Delta x$ equal to 0

14: $\quad x=6$ // Assign 6 to $x$

15: elseif $\Delta y$ less than 0 and $\Delta x$ greater than 0

16: $x=7 \quad / /$ Assign 7 to $x$

$17:$ else

18: $x=0$ // Assign 0 to $x$

\section{Results}

In determining the feature vector that are suitable for the classification of the fish activities, the movement directions were extracted using Freeman Chain Code. The set of numbers generated through the use of chain code was converted into FD for extracting the pattern signature of a given activity. The chain code plots and magnitude plots of the FD for the feeding and escape movements is shown in Fig. 2 (a) and (b) respectively. By observing this Figure (Fig. 2 (a) and (b)), FD give pattern that are more compact and easier to match than Chain Code. Therefore, it was view that using FD to classify fish activities could prove useful than Chain Code.
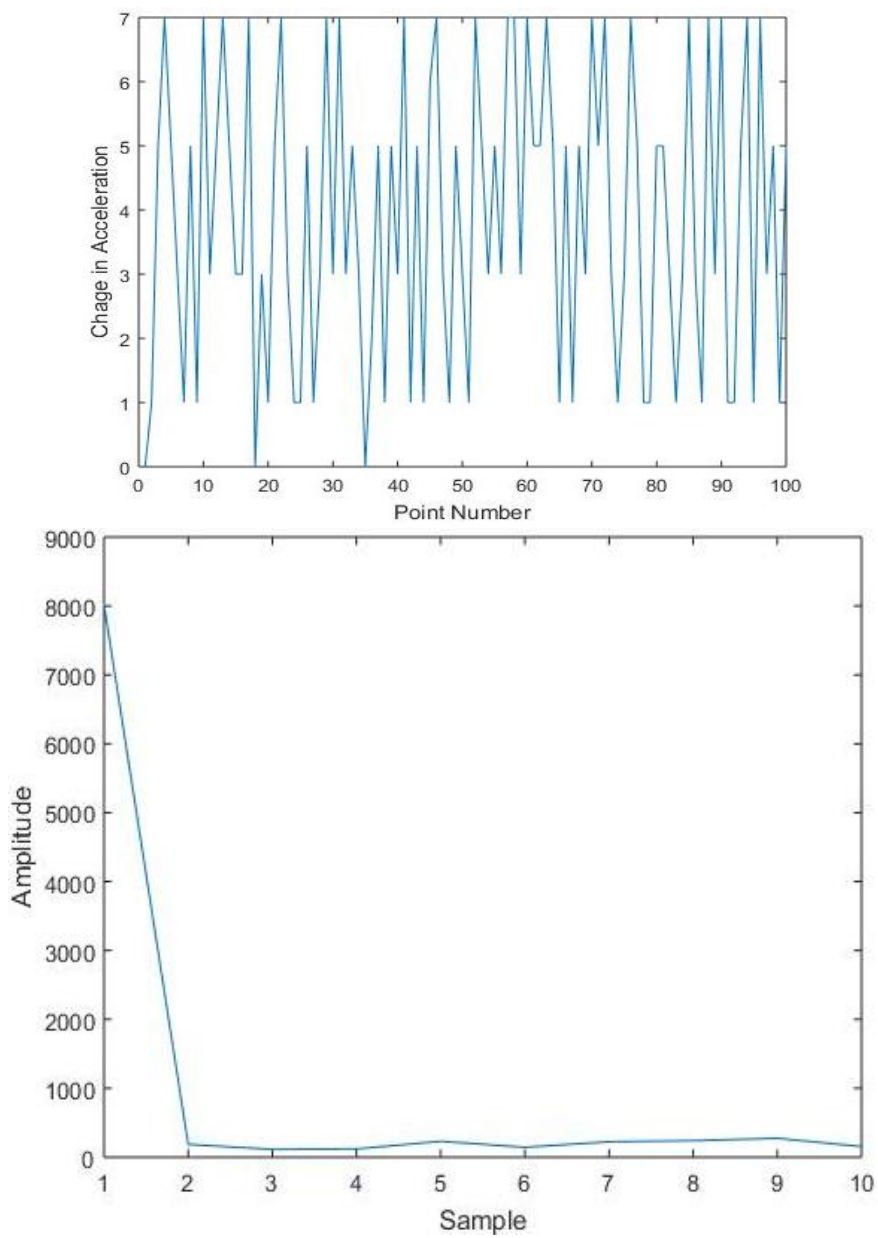

(a) 

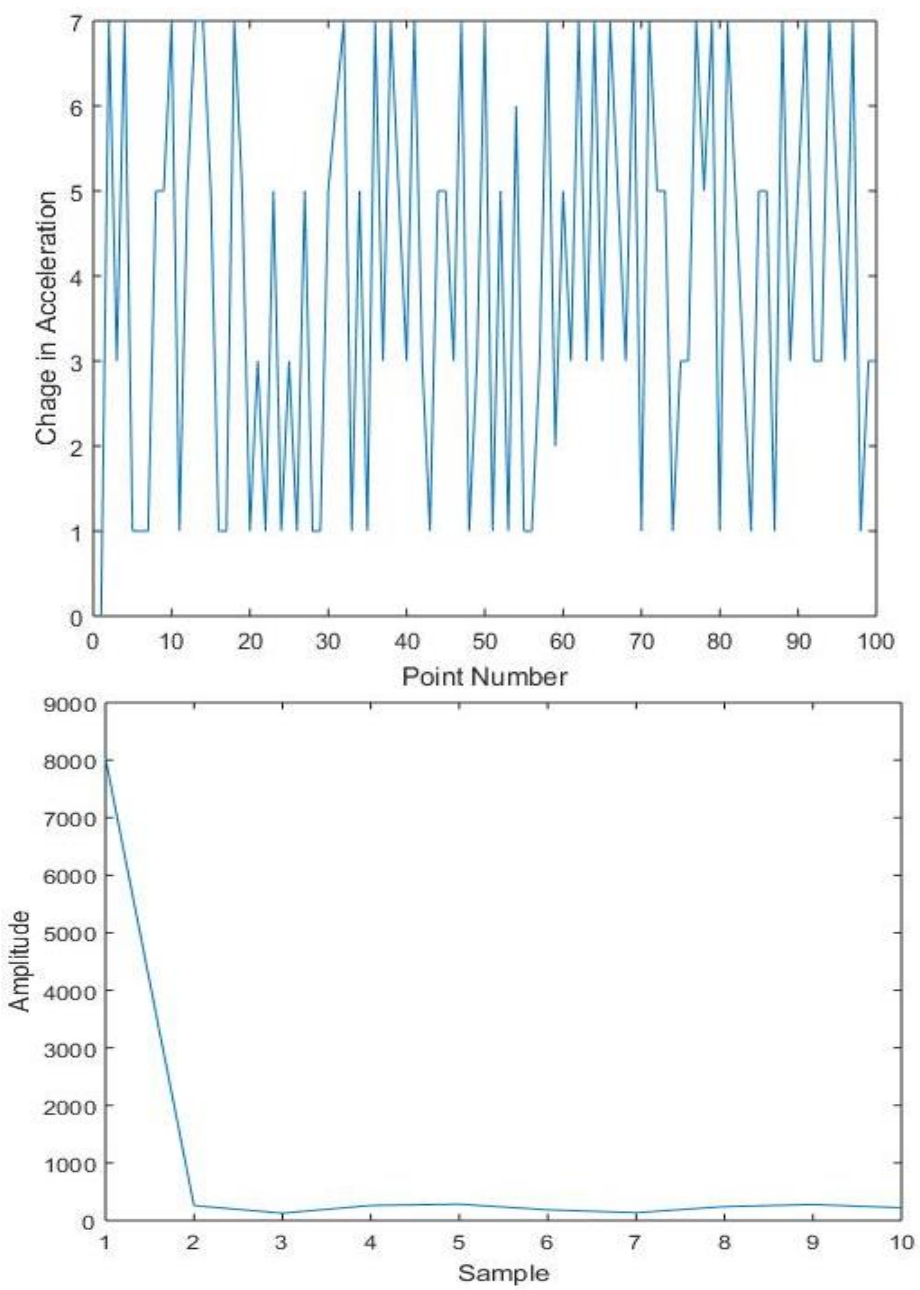

(b)

Figure 2. Chain code plots and FD of the (a) Feeding and (b) Escape movement

\section{Conclusion}

The development of a vibration based fish feeding regime system using vibration analysis has been presented in this paper. This was accomplished through the extraction of signals from accelerometer for the escape and feeding activities. In determining the feature vector suitable for the individual activity recognition, the movements directions were extracted using developed 8-directional Chain Codes generator algorithm for the computation of Chain Code represent movement direction of the fishes. The set of sequence features obtained was further processed using DFT in analyzing the movement pattern. The FD obtained from the Chain Code and movement direction were then used for the developed system.

\section{Acknowledgements}

The authors would like to acknowledge Takuji, N., Yuuki, K., Nobuki, A., Hiromichi, M., and Shun, W. for permit reuse of their dataset. The help provided by my colleague, Ajao, Lukman Adewale in collecting the relevant references is also gratefully acknowledged. 


\section{References}

[1] R. Subasinghe, D. Soto, and J. Jia, "Global aquaculture and its role in sustainable development," Reviews in Aquaculture, vol.1, no.1, pp.2-9, (2009)

[2] R. L. Naylor et al., "Effect of aquaculture on world fish supplies," Nature, vol.405, no.6790, p.1017, (2000)

[3] M. Jamal, "Modeling and control of the fish feeder system," Project report. Faculty of Mechanical and Manufacturing Engineering, University Tun Hussein Onn Malaysia, (2013)

[4] H. S. AlZubi, W. Al-Nuaimy, J. Buckley, and I. Young, "An intelligent behavior-based fish feeding system," in 2016 13th International Multi-Conference on Systems, Signals \& Devices (SSD), pp.22-29, (2016) DOI: 10.1109/SSD.2016.7473754

[5] J.-V. Lee, J.-L. Loo, Y.-D. Chuah, P.-Y. Tang, Y.-C. Tan, and W.-J. Goh, "The use of vision in a sustainable aquaculture feeding system”, Research Journal of Applied Sciences, Engineering and Technology, vol.6, no.19, pp.3658-3669, (2013)

[6] A. W. Fast, T. Qin, and J. P. Szyper, "A new method for assessing fish feeding rhythms using demand feeders and automated data acquisition," Aquacultural engineering, vol.16, no.4, pp.213-220, (1997) DOI: 10.1016/s0144-8609(97)00003-4

[7] Y. Atoum, S. Srivastava, and X. Liu, "Automatic feeding control for dense aquaculture fish tanks," IEEE Signal Processing Letters, vol.22, no.8, pp.1089-1093, (2014) DOI: 10.1109/LSP.2014.2385794

[8] K. R. Skøien and J. A. Alfredsen, "Feeding of large-scale fish farms: Motion characterization of a pneumatic rotor feed spreader," in 2014 Oceans-St. John's, pp.1-7, (2014) DOI: 10.1109/OCEANS.2014.7003103

[9] M. Masser and D. Cline, "Caged fish production in Alabama," Extension Aquaculturist, ANR-957, Alabama University, (1990)

[10] D. P. Bureau, K. Hua, and C. Y. Cho, "Effect of feeding level on growth and nutrient deposition in rainbow trout (Oncorhynchus mykiss Walbaum) growing from 150 to 600 g," Aquaculture research, vol.37, no.11, pp.1090-1098, (2006) DOI: 10.1111/j.1365-2109.2006.01532.x

[11] J. Solomon and M. Ezigbo, "Polyculture of Heteroclarias/Tilapia under different feeding regimes," New York Science Journal, vol.3, no.10, pp.42-57, (2010)

[12] C. Y. Cho, "Development of computer models for fish feeding standards and aquaculture waste estimations: A treatise," in SIMPOSIO INTERNACIONAL DE NUTRICIÓN ACUÍCOLA, vol.7, pp.375-395, (2004)

[13] D. T. Ani, M. G. F. Cueto, N. J. G. Diokno, and K. R. R. Perez, "Solar powered automatic shrimp feeding system," Asia Pacific Journal of Multidisciplinary Research, vol.3, no.5, (2015)

[14] A. O. OGUNLELA, "Development and performance evaluation of an Automatic Fish Feeder," in 2014 Montreal, Quebec Canada July 13-16, pp.1, (2014)

[15] M. Noor, A. Hussian, M. Saaid, M. Ali, and M. Zolkapli, "The design and development of automatic fish feeder system using PIC microcontroller," in 2012 IEEE Control and System Graduate Research Colloquium, pp.343347, (2012) DOI: 10.1109/ICSGRC.2012.6287189

[16] S. E. Abdallah and W. M. Elmessery, "An automatic feeder with two different control systems for intensive mirror carp production," Journal of Agricultural Engineering and Biotechnology, vol.2, no.3, pp.36, (2014)

[17] M. Niswar, Z. Zainuddin, Y. Fujaya, and Z. M. Putra, "An automated feeding system for soft shell crab," Indonesian Journal of Electrical Engineering and Computer Science, vol.5, no.3, pp.564-568, (2017)

[18] Y. Yin, L. Chen, Y. Xu, J. Wan, H. Zhang, and Z. Mai, "QoS prediction for service recommendation with deep feature learning in edge computing environment," Mobile Networks and Applications, pp.1-11, (2019) DOI: 10.1007/s11036-019-01241-7

[19] C. Chang, W. Fang, R.-C. Jao, C. Shyu, and I. Liao, "Development of an intelligent feeding controller for indoor intensive culturing of eel," Aquacultural engineering, vol.32, no.2, pp.343-353, (2005) DOI: 10.1016/j.aquaeng.2004.07.004 
Intelligent Fish Feeding Regime System using Vibration Analysis

[20] N. Papandroulakis, G. Markakis, P. Divanach, and M. Kentouri, "Feeding requirements of sea bream (Sparus aurata) larvae under intensive rearing conditions: Development of a fuzzy logic controller for feeding," Aquacultural Engineering, vol.21, no.4, pp.285-299, (2000) DOI: 10.1016/S0144-8609(99)00036-9

[21] S. D. Rana and S. Rani, "Fuzzy logic based control system for fresh water aquaculture: A matlab based simulation approach," Serbian Journal of Electrical Engineering, vol.12, no.2, pp.171-182, (2015) DOI: 10.2298/SJEE1502171R

[22] T. Noda, Y. Kawabata, N. Arai, H. Mitamura, and S. Watanabe, "Monitoring escape and feeding behaviours of cruiser fish by inertial and magnetic sensors," PLoS One, vol.8, no.11, (2013) DOI: 10.1371/journal. pone.0079392

[23] T. V. Gopal and V. Prasad, "A novel approach to shape based image retrieval integrating adapted fourier descriptors and freeman code,” Int. J. Comput. Sci. Netw. Security, vol.8, no.6, pp.293-302, (2008) 